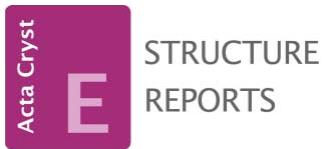

OPEN $\curvearrowright$ ACCESS

ISSN 1600-5368

\section{Crystal structure of azido $\left(\eta^{5}\right.$-cyclopenta- dienyl)bis(triphenylphosphane- $\boldsymbol{*} P$ )- ruthenium(II) dichloromethane hemisolvate}

\author{
Adriana Hernández-Calva, ${ }^{a}$ Lidia Meléndez-Balbuena, ${ }^{b}$ \\ Maribel Arroyo ${ }^{\mathrm{a}}$ and Armando Ramírez-Monroy ${ }^{\mathrm{a} *}$ \\ ${ }^{a}$ Centro de Química del Instituto de Ciencias, Benemérita Universidad Autónoma de \\ Puebla, Ciudad Universitaria, San Manuel, 72570, Puebla, Puebla, Mexico, and \\ ${ }^{\mathbf{b}}$ Facultad de Ciencias Químicas, Benemérita Universidad Autónoma de Puebla, \\ Ciudad Universitaria, San Manuel, 72570, Puebla, Puebla, Mexico. *Correspon- \\ dence e-mail: armando.ramirez@correo.buap.mx
}

Received 15 August 2014; accepted 25 August 2014

Edited by M. Weil, Vienna University of Technology, Austria

The title solvated complex, $\left[\mathrm{Ru}\left(\eta^{5}-\mathrm{C}_{5} \mathrm{H}_{5}\right)\left(\mathrm{N}_{3}\right)\left\{\mathrm{P}\left(\mathrm{C}_{6} \mathrm{H}_{5}\right)_{3}\right\}_{2}\right] \cdot-$ $0.5 \mathrm{CH}_{2} \mathrm{Cl}_{2}$, displays a typical piano-stool geometry about the $\mathrm{Ru}^{\mathrm{II}}$ atom. The bond lengths and angles of the cyclopentadienyl and phosphane ligands are very similar to that of the unsolvated complex [Taqui Khan et al. (1994). Acta Cryst. C50, 502-504]. The azide anion displays similar $\mathrm{N}-\mathrm{N}$ distances of 1.173 (3) and 1.156 (3) $\AA$ and has an $\mathrm{N}-\mathrm{N}-\mathrm{Ru}$ angle of $119.20(15)^{\circ}$, indicating a greater contribution of the canonical form $\mathrm{Ru}-\mathrm{N}=\mathrm{N}^{(+)}=\mathrm{N}^{(-)}$for the bonding situation. An intramolecular $\mathrm{C}-\mathrm{H} \cdots \mathrm{N}$ hydrogen-bonding interaction between one ortho $\mathrm{H}$ atom of a phosphane ligand and the $\mathrm{N}$ atom coordinating to the metal is observed. A similar intermolecular interaction is observed between a meta $\mathrm{H}$ atom of a phosphane ligand and the terminal azide $\mathrm{N}$ atom of a neighbouring complex. Finally, two $\mathrm{C}-\mathrm{H} \cdots \mathrm{N}$ interactions exists between the $\mathrm{H}$ atoms of the dichloromethane solvent molecule and the terminal $\mathrm{N}$ atom of two azide anions. The solvent molecule is located about a twofold rotation axis and shows disorder of the $\mathrm{Cl}$ atoms with an occupancy ratio of 0.62 (3):0.38 (3).

Keywords: crystal structure; ruthenium; azido complex; piano-stool geometry.

CCDC reference: 1021189

\section{Related literature}

The structure of the unsolvated ruthenium(II) complex was determined by Taqui Khan et al. (1994). For other azide ruthenium(II) complexes, see: Moura et al. (1999); Govinda- swamy et al. (2005). For metal azide chemistry, see: Fehlhammer \& Beck (2013); Seok \& Klapötke (2010). Nonclassical hydrogen bonds were assigned on basis of distances that are shorter than the sum of the van der Waals radii (Bondi, 1964) of respective atoms. For synthetic details, see: Moura et al. (2002).

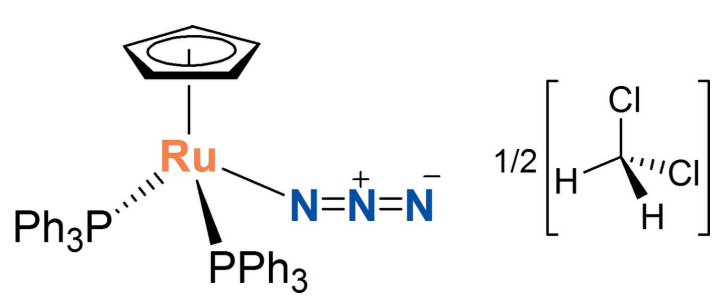

\section{Experimental}

\subsection{Crystal data}

$\left[\mathrm{Ru}\left(\mathrm{C}_{5} \mathrm{H}_{5}\right)\left(\mathrm{N}_{3}\right)\left(\mathrm{C}_{18} \mathrm{H}_{15} \mathrm{P}\right)_{2}\right] \cdot-$ $0.5 \mathrm{CH}_{2} \mathrm{Cl}_{2}$

$M_{r}=775.19$

Monoclinic, $I 2 / a$

$a=20.1817$ (4) §

$b=12.4559(3) \AA$

$c=28.6781(6) \AA$

$$
\begin{aligned}
& \beta=94.213(2)^{\circ} \\
& V=7189.7(3) \AA^{3} \\
& Z=8 \\
& \text { Mo } K \alpha \text { radiation } \\
& \mu=0.63 \mathrm{~mm}^{-1} \\
& T=293 \mathrm{~K} \\
& 0.72 \times 0.51 \times 0.20 \mathrm{~mm}
\end{aligned}
$$

\subsection{Data collection}

Agilent Xcalibur Atlas Gemini diffractometer

Absorption correction: analytical [CrysAlis PRO (Agilent, 2012) using a multi=faceted crystal model based on expressions

derived by Clark \& Reid (1995)]

$T_{\min }=0.737, T_{\max }=0.897$ 36924 measured reflections 7106 independent reflections 6038 reflections with $I>2 \sigma(I)$ $R_{\text {int }}=0.032$

\subsection{Refinement}

$R\left[F^{2}>2 \sigma\left(F^{2}\right)\right]=0.027$

$w R\left(F^{2}\right)=0.065$

$S=1.06$

7106 reflections

449 parameters

$\mathrm{H}$-atom parameters constrained

$\Delta \rho_{\max }=0.33{\mathrm{e} \AA^{-3}}^{-3}$

$\Delta \rho_{\min }=-0.49 \mathrm{e} \AA^{-3}$

Table 1

Hydrogen-bond geometry $\left(\AA,^{\circ}\right)$.

\begin{tabular}{lllll}
\hline$D-\mathrm{H} \cdots A$ & $D-\mathrm{H}$ & $\mathrm{H} \cdots A$ & $D \cdots A$ & $D-\mathrm{H} \cdots A$ \\
\hline $\mathrm{C} 18-\mathrm{H} 18 \cdots \mathrm{N} 1$ & 0.93 & 2.35 & $3.204(3)$ & 153 \\
$\mathrm{C} 23-\mathrm{H} 23 \cdots \mathrm{N} 3^{\mathrm{i}}$ & 0.93 & 2.62 & $3.537(4)$ & 167 \\
$\mathrm{C}^{2} 2-\mathrm{H} 42 A \cdots \mathrm{N} 3^{\mathrm{ii}}$ & 0.97 & 2.4 & $3.338(4)$ & 162 \\
$\mathrm{C}^{2}-\mathrm{H} 42 B \cdots \mathrm{N} 3^{\mathrm{i}}$ & 0.97 & 2.4 & $3.338(4)$ & 162
\end{tabular}

Symmetry codes: (i) $x+\frac{1}{2},-y+1, z$; (ii) $-x+1,-y+1,-z+1$.

Data collection: CrysAlis PRO (Agilent, 2012); cell refinement: CrysAlis PRO; data reduction: CrysAlis RED (Agilent, 2012); program(s) used to solve structure: OLEX2 (Dolomanov et al., 2009); program(s) used to refine structure: SHELXL2014 (Sheldrick, 2008); molecular graphics: ORTEP-3 for Windows (Farrugia, 2012); software used to prepare material for publication: WinGX (Farrugia, 2012). 


\section{Acknowledgements}

We gratefully acknowledge VIEP (ARCS-NAT-14 G) for financial support. AHC thanks CONACYT for a doctoral fellowship.

Supporting information for this paper is available from the IUCr electronic archives (Reference: WM5050).

\section{References}

Agilent (2012). CrysAlis PRO and CrysAlis RED. Agilent Technologies Ltd, Yarnton, Oxfordshire, England.
Bondi, A. (1964). J. Phys. Chem. 68, 441-451.

Clark, R. C. \& Reid, J. S. (1995). Acta Cryst. A51, 887-897.

Dolomanov, O. V., Bourhis, L. J., Gildea, R. J., Howard, J. A. K. \& Puschmann, H. (2009). J. Appl. Cryst. 42, 339-341.

Farrugia, L. J. (2012). J. Appl. Cryst. 45, 849-854.

Fehlhammer, W. P. \& Beck, W. (2013). Z. Anorg. Allg. Chem. 639, 1053-1082.

Govindaswamy, P., Carroll, P. J., Mozharivskyj, Y. A. \& Kollipara, M. R. (2005). J. Organomet. Chem. 690, 885-894.

Moura, E. M., Dickman, M. H., Siebald, H. G. L. \& Gama, G. J. (1999). Polyhedron, 18, 2899-2906.

Moura, E. M., Siebald, H. G. L. \& de Lima, G. M. (2002). Polyhedron, 21, 2323-2331.

Seok, W. K. \& Klapötke, T. M. (2010). Bull. Korean Chem. Soc. 31, 781-788.

Sheldrick, G. M. (2008). Acta Cryst. A64, 112-122.

Taqui Khan, M. M., Bhadbhade, M. M., Siddiqui, M. R. H., Venkatasubramanian, K. \& Tikhonova, J. A. (1994). Acta Cryst. C50, 502-504. 


\section{supporting information}

Acta Cryst. (2014). E70, m345-m346 [doi:10.1107/S1600536814019187]

\section{Crystal structure of azido $\left(\eta^{5}\right.$-cyclopentadienyl)bis(triphenylphosphane- $\kappa P$ )ruthenium(II) dichloromethane hemisolvate}

\section{Adriana Hernández-Calva, Lidia Meléndez-Balbuena, Maribel Arroyo and Armando Ramírez- Monroy}

\section{S1. Synthesis and crystallization}

The title compound was synthesized following a slightly modified procedure developed by Moura et al. (2002). Under a dry nitrogen atmosphere, to $\left[\mathrm{Ru}\left(\eta 5-\mathrm{C}_{5} \mathrm{H}_{5}\right)\left(\mathrm{PPh}_{3}\right)_{2} \mathrm{Cl}\right](0.100 \mathrm{~g}, 0.138 \mathrm{mmol})$ dissolved in $20 \mathrm{ml}$ of dry ethanol was added $\mathrm{NaN}_{3}(0.134 \mathrm{~g}, 2.061 \mathrm{mmol})$. The stirred mixture was refluxed for $6 \mathrm{~h}$. After this time, the solvent was removed under vacuum and the product was extracted with $4 \mathrm{ml}$ of dry dichloromethane. To the resulting orange solution, $8 \mathrm{ml}$ of degassed hexanes were added in order to induce crystallization at $281 \mathrm{~K}$. Red-orange colored crystals of the title compound were obtained in 82\% yield. M.p. $393 \mathrm{~K}$ (dec.); IR (KBr) $v\left(\mathrm{~N}_{3}\right) 2023 \mathrm{~cm}^{-1}$.

\section{S2. Refinement}

All hydrogen atoms were generated at calculated positions with $\mathrm{C}-\mathrm{H}$ distances constrained to $0.93-0.97 \AA$. All hydrogen atoms were refined using a riding model approximation with $U_{\text {iso }}(\mathrm{H})=1.2 U_{\text {eq }}(\mathrm{C})$. The dichloromethane molecule is located about a twofold rotation axis. Its chlorine atoms are disordered over two positions, with refined occupancies of 0.62 (3) and $0.38(3)$. 

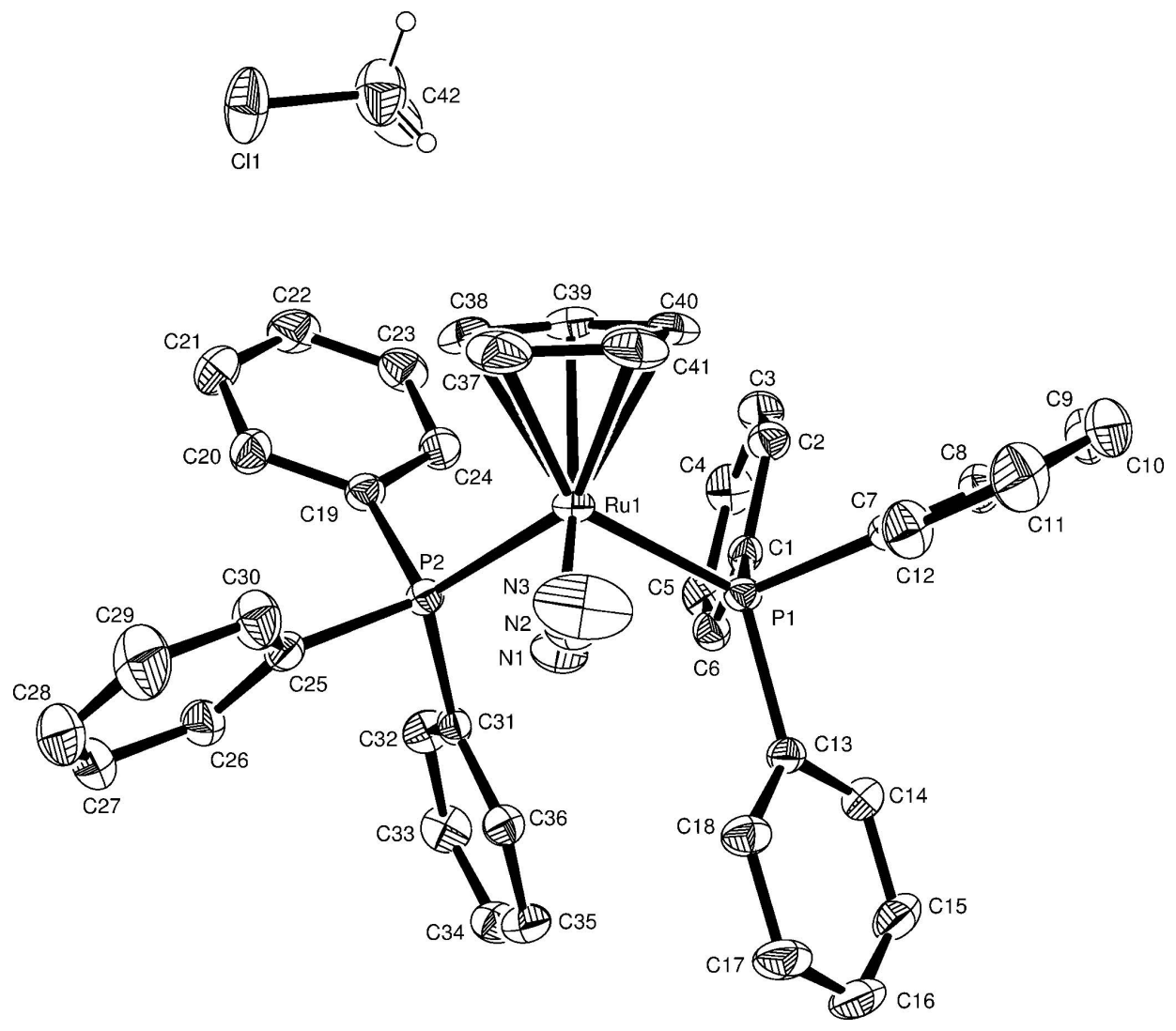

\section{Figure 1}

Molecular structure of the title compound showing the atom-numbering scheme. Displacement ellipsoids are drawn at the $30 \%$ probability level. Only the major component of the disordered dichloromethane solvate is shown. Hydrogen atoms of the metal complex have been removed for clarity. 


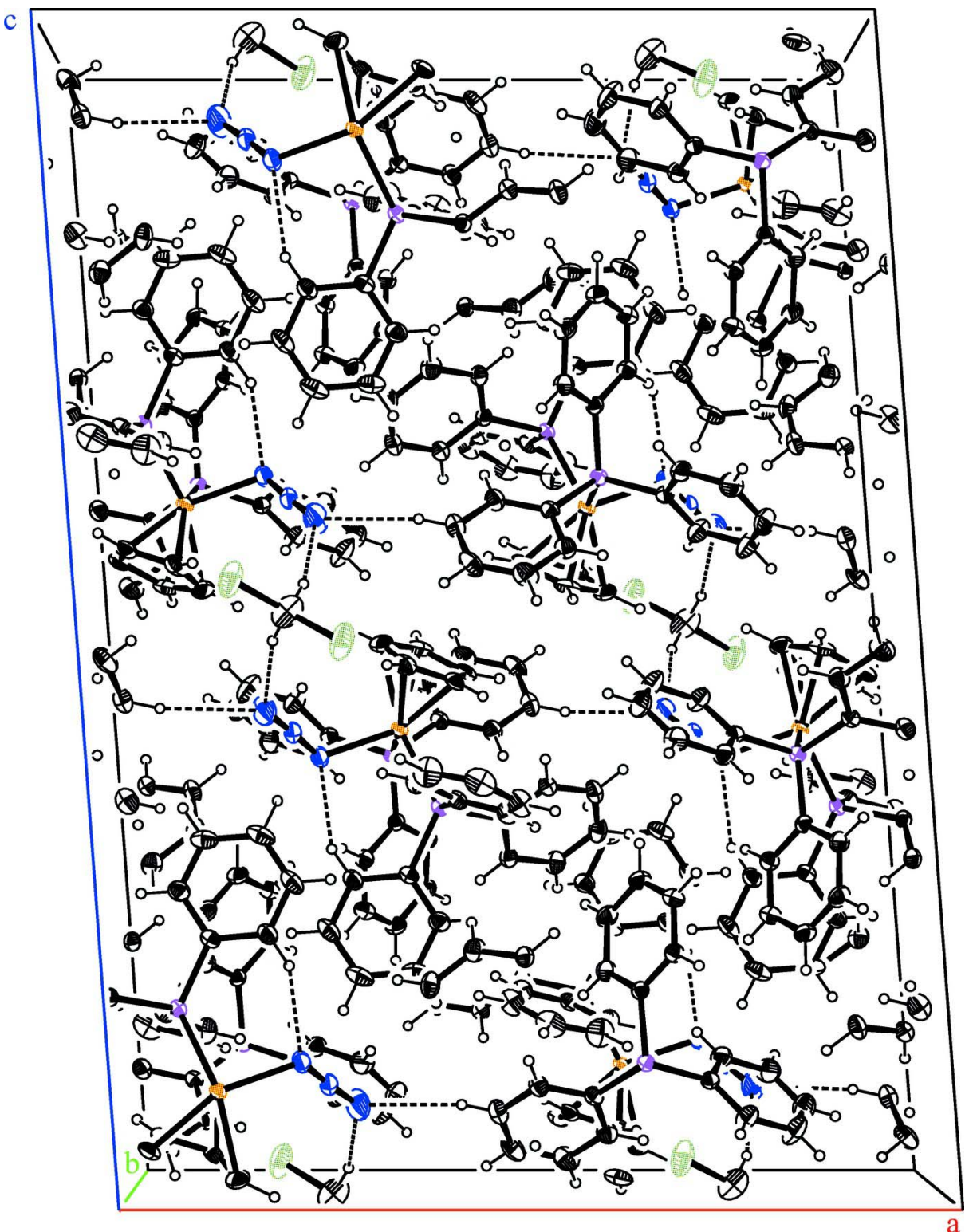

Figure 2

View of the molecular arrangement in the title structure viewed along [010]. Hydrogen bonds are denoted by dashed lines.

Azido $\left(\eta^{5}\right.$-cyclopentadienyl)bis(triphenylphosphane- $\kappa$ P)ruthenium(II) dichloromethane hemisolvate

Crystal data

$\left[\mathrm{Ru}\left(\mathrm{C}_{5} \mathrm{H}_{5}\right)\left(\mathrm{N}_{3}\right)\left(\mathrm{C}_{18} \mathrm{H}_{15} \mathrm{P}\right)_{2}\right] \cdot 0.5 \mathrm{CH}_{2} \mathrm{Cl}_{2}$

$M_{r}=775.19$

Monoclinic, $I 2 / a$

$a=20.1817(4) \AA$

$b=12.4559(3) \AA$

$c=28.6781(6) \AA$

$\beta=94.213(2)^{\circ}$

$V=7189.7(3) \AA^{3}$

$Z=8$
$F(000)=3176$

$D_{\mathrm{x}}=1.432 \mathrm{Mg} \mathrm{m}^{-3}$

Mo $K \alpha$ radiation, $\lambda=0.71073 \AA$

Cell parameters from 13778 reflections

$\theta=3.5-29.5^{\circ}$

$\mu=0.63 \mathrm{~mm}^{-1}$

$T=293 \mathrm{~K}$

Prism, orange

$0.72 \times 0.51 \times 0.20 \mathrm{~mm}$ 


\section{Data collection}

Agilent Xcalibur Atlas Gemini diffractometer

Radiation source: Enhance (Mo) X-ray Source Graphite monochromator

Detector resolution: 10.5564 pixels $\mathrm{mm}^{-1}$

$\omega$ scans

Absorption correction: analytical

[CrysAlis PRO (Agilent, 2012) using a

multi=faceted crystal model based on

expressions derived by Clark \& Reid (1995)]

\section{Refinement}

Refinement on $F^{2}$

Least-squares matrix: full

$R\left[F^{2}>2 \sigma\left(F^{2}\right)\right]=0.027$

$w R\left(F^{2}\right)=0.065$

$S=1.06$

7106 reflections

449 parameters

0 restraints
$T_{\min }=0.737, T_{\max }=0.897$

36924 measured reflections

7106 independent reflections

6038 reflections with $I>2 \sigma(I)$

$R_{\text {int }}=0.032$

$\theta_{\max }=26.1^{\circ}, \theta_{\min }=3.3^{\circ}$

$h=-24 \rightarrow 24$

$k=-15 \rightarrow 15$

$l=-35 \rightarrow 35$

Hydrogen site location: inferred from neighbouring sites

$\mathrm{H}$-atom parameters constrained

$w=1 /\left[\sigma^{2}\left(F_{0}^{2}\right)+(0.022 P)^{2}+9.5067 P\right]$

where $P=\left(F_{\mathrm{o}}{ }^{2}+2 F_{\mathrm{c}}{ }^{2}\right) / 3$

$(\Delta / \sigma)_{\max }=0.001$

$\Delta \rho_{\max }=0.33 \mathrm{e} \AA^{-3}$

$\Delta \rho_{\min }=-0.49 \mathrm{e} \AA^{-3}$

Extinction correction: SHELXL

Extinction coefficient: 0.00036 (4)

Special details

Experimental. none

Geometry. All e.s.d.'s (except the e.s.d. in the dihedral angle between two 1.s. planes) are estimated using the full covariance matrix. The cell e.s.d.'s are taken into account individually in the estimation of e.s.d.'s in distances, angles and torsion angles; correlations between e.s.d.'s in cell parameters are only used when they are defined by crystal symmetry. An approximate (isotropic) treatment of cell e.s.d.'s is used for estimating e.s.d.'s involving 1.s. planes.

Refinement. None

Fractional atomic coordinates and isotropic or equivalent isotropic displacement parameters $\left(\AA^{2}\right)$

\begin{tabular}{llllll}
\hline & $x$ & $y$ & $z$ & $U_{\text {iso }} / U_{\text {eq }}$ & Occ. $(<1)$ \\
\hline C1 & $0.49378(9)$ & $0.46227(15)$ & $0.32350(7)$ & $0.0298(4)$ & \\
C2 & $0.55095(10)$ & $0.44269(18)$ & $0.35275(8)$ & $0.0385(5)$ & \\
H2 & 0.5499 & 0.3909 & 0.3761 & $0.046^{*}$ & \\
C3 & $0.60895(11)$ & $0.4986(2)$ & $0.34772(9)$ & $0.0471(6)$ & \\
H3 & 0.6466 & 0.4837 & 0.3673 & $0.057^{*}$ & \\
C4 & $0.61134(12)$ & $0.5763(2)$ & $0.31387(10)$ & $0.0519(6)$ & \\
H4 & 0.6505 & 0.6138 & 0.3104 & $0.062^{*}$ & \\
C5 & $0.55533(12)$ & $0.59808(19)$ & $0.28509(9)$ & $0.0483(6)$ & \\
H5 & 0.5567 & 0.651 & 0.2623 & $0.058^{*}$ & \\
C6 & $0.49689(11)$ & $0.54182(16)$ & $0.28979(8)$ & $0.0363(5)$ & \\
H6 & 0.4594 & 0.5575 & 0.2702 & $0.044^{*}$ & \\
C7 & $0.43752(10)$ & $0.25218(16)$ & $0.34102(7)$ & $0.0344(5)$ & \\
C8 & $0.49774(12)$ & $0.20901(19)$ & $0.33053(9)$ & $0.0461(6)$ & \\
H8 & 0.5292 & 0.2527 & 0.3178 & $0.055^{*}$ & \\
C9 & $0.51178(15)$ & $0.1019(2)$ & $0.33864(11)$ & $0.0608(7)$ & \\
H9 & 0.5529 & 0.0743 & 0.332 & $0.073^{*}$ &
\end{tabular}




\begin{tabular}{|c|c|c|c|c|}
\hline $\mathrm{C} 10$ & $0.46576(18)$ & $0.0364(2)$ & $0.35632(11)$ & $0.0688(9)$ \\
\hline H10 & 0.4758 & -0.0353 & 0.3625 & $0.083 *$ \\
\hline C11 & $0.40434(17)$ & $0.0766(2)$ & $0.36496(12)$ & $0.0711(9)$ \\
\hline H11 & 0.3722 & 0.0313 & 0.3758 & $0.085^{*}$ \\
\hline $\mathrm{C} 12$ & $0.39027(13)$ & 0.18415 (19) & $0.35761(10)$ & $0.0525(6)$ \\
\hline H12 & 0.3488 & 0.211 & 0.3638 & $0.063 *$ \\
\hline $\mathrm{C} 13$ & $0.37121(10)$ & $0.38670(16)$ & $0.27572(7)$ & $0.0320(4)$ \\
\hline C14 & $0.40484(12)$ & $0.37479(18)$ & $0.23548(8)$ & $0.0431(5)$ \\
\hline H14 & 0.451 & 0.374 & 0.2376 & $0.052 *$ \\
\hline $\mathrm{C} 15$ & $0.37038(14)$ & $0.3640(2)$ & $0.19232(9)$ & $0.0540(7)$ \\
\hline H15 & 0.3935 & 0.3564 & 0.1656 & $0.065^{*}$ \\
\hline $\mathrm{C} 16$ & $0.30208(15)$ & $0.3646(2)$ & $0.18854(9)$ & $0.0582(7)$ \\
\hline H16 & 0.279 & 0.3588 & 0.1594 & $0.07 *$ \\
\hline $\mathrm{C} 17$ & $0.26818(13)$ & $0.3737(2)$ & $0.22806(9)$ & $0.0554(7)$ \\
\hline H17 & 0.222 & 0.3728 & 0.2256 & $0.066^{*}$ \\
\hline C18 & $0.30229(11)$ & $0.38420(18)$ & $0.27166(8)$ & $0.0422(5)$ \\
\hline H18 & 0.2788 & 0.3896 & 0.2983 & $0.051^{*}$ \\
\hline $\mathrm{C} 19$ & $0.42371(10)$ & $0.72523(17)$ & $0.41116(7)$ & $0.0350(5)$ \\
\hline $\mathrm{C} 20$ & $0.40982(12)$ & 0.79993 (19) & $0.44478(8)$ & $0.0454(6)$ \\
\hline $\mathrm{H} 20$ & 0.366 & 0.8123 & 0.4514 & $0.054^{*}$ \\
\hline $\mathrm{C} 21$ & $0.46075(14)$ & $0.8563(2)$ & $0.46864(10)$ & $0.0589(7)$ \\
\hline $\mathrm{H} 21$ & 0.4509 & 0.9064 & 0.4912 & $0.071^{*}$ \\
\hline $\mathrm{C} 22$ & $0.52559(14)$ & $0.8392(2)$ & $0.45935(10)$ & $0.0585(7)$ \\
\hline $\mathrm{H} 22$ & 0.5595 & 0.8771 & 0.4757 & $0.07 *$ \\
\hline $\mathrm{C} 23$ & $0.54049(12)$ & $0.7659(2)$ & $0.42584(9)$ & $0.0516(6)$ \\
\hline $\mathrm{H} 23$ & 0.5844 & 0.7547 & 0.4192 & $0.062 *$ \\
\hline $\mathrm{C} 24$ & $0.48992(11)$ & $0.70906(19)$ & $0.40198(8)$ & $0.0433(5)$ \\
\hline $\mathrm{H} 24$ & 0.5002 & 0.6592 & 0.3795 & $0.052^{*}$ \\
\hline $\mathrm{C} 25$ & $0.28225(10)$ & $0.70450(18)$ & $0.39722(7)$ & $0.0360(5)$ \\
\hline $\mathrm{C} 26$ & $0.26325(12)$ & $0.8048(2)$ & $0.37980(9)$ & $0.0479(6)$ \\
\hline $\mathrm{H} 26$ & 0.2895 & 0.8397 & 0.3592 & $0.058^{*}$ \\
\hline $\mathrm{C} 27$ & $0.20570(14)$ & $0.8534(2)$ & $0.39266(10)$ & $0.0627(8)$ \\
\hline $\mathrm{H} 27$ & 0.1937 & 0.9208 & 0.3809 & $0.075^{*}$ \\
\hline $\mathrm{C} 28$ & $0.16642(15)$ & $0.8026(3)$ & $0.42271(11)$ & $0.0722(9)$ \\
\hline H28 & 0.1277 & 0.8351 & 0.4314 & $0.087^{*}$ \\
\hline $\mathrm{C} 29$ & $0.18428(16)$ & $0.7042(3)$ & $0.43981(12)$ & $0.0785(10)$ \\
\hline H29 & 0.1575 & 0.6696 & 0.4601 & $0.094 *$ \\
\hline $\mathrm{C} 30$ & $0.24230(13)$ & $0.6544(2)$ & $0.42730(9)$ & $0.0551(7)$ \\
\hline H30 & 0.254 & 0.5872 & 0.4394 & $0.066^{*}$ \\
\hline $\mathrm{C} 31$ & $0.35924(10)$ & $0.68670(16)$ & $0.32038(7)$ & $0.0307(4)$ \\
\hline $\mathrm{C} 32$ & $0.39666(11)$ & 0.77203 (19) & $0.30534(8)$ & $0.0438(5)$ \\
\hline H32 & 0.4261 & 0.8076 & 0.3265 & $0.053^{*}$ \\
\hline C33 & $0.39017(12)$ & $0.8041(2)$ & $0.25902(9)$ & $0.0498(6)$ \\
\hline H33 & 0.4145 & 0.8625 & 0.2494 & $0.06^{*}$ \\
\hline C34 & $0.34825(12)$ & $0.7507(2)$ & $0.22735(8)$ & $0.0493(6)$ \\
\hline H34 & 0.3449 & 0.7716 & 0.1961 & $0.059^{*}$ \\
\hline C35 & $0.31111(12)$ & $0.6662(2)$ & $0.24174(8)$ & $0.0471(6)$ \\
\hline H35 & 0.2828 & 0.6295 & 0.2202 & $0.057^{*}$ \\
\hline
\end{tabular}




\begin{tabular}{|c|c|c|c|c|c|}
\hline $\mathrm{C} 36$ & $0.31569(11)$ & $0.63574(17)$ & $0.28805(7)$ & $0.0368(5)$ & \\
\hline $\mathrm{H} 36$ & 0.2891 & 0.5802 & 0.2977 & $0.044 *$ & \\
\hline $\mathrm{C} 37$ & $0.35806(14)$ & $0.4319(3)$ & $0.47670(9)$ & $0.0638(8)$ & \\
\hline $\mathrm{H} 37$ & 0.3159 & 0.4377 & 0.4914 & $0.077^{*}$ & \\
\hline $\mathrm{C} 38$ & $0.40514(15)$ & $0.5138(2)$ & $0.47330(8)$ & $0.0561(7)$ & \\
\hline H38 & 0.4015 & 0.5871 & 0.4854 & $0.067^{*}$ & \\
\hline C39 & $0.45983(12)$ & $0.4716(2)$ & $0.45186(8)$ & $0.0482(6)$ & \\
\hline H39 & 0.5012 & 0.5096 & 0.4469 & $0.058^{*}$ & \\
\hline $\mathrm{C} 40$ & $0.44716(12)$ & $0.3625(2)$ & $0.44317(8)$ & $0.0478(6)$ & \\
\hline $\mathrm{H} 40$ & 0.478 & 0.3118 & 0.4301 & $0.057^{*}$ & \\
\hline $\mathrm{C} 41$ & $0.38453(14)$ & $0.3368(2)$ & $0.45750(9)$ & $0.0576(7)$ & \\
\hline $\mathrm{H} 41$ & 0.3639 & 0.2655 & 0.4566 & $0.069^{*}$ & \\
\hline $\mathrm{C} 42$ & 0.75 & 0.8824 & 0.5 & $0.0924(16)$ & \\
\hline $\mathrm{H} 42 \mathrm{~A}$ & 0.7713 & 0.8364 & 0.5239 & $0.111^{*}$ & 0.5 \\
\hline H42B & 0.7287 & 0.8364 & 0.4761 & $0.111^{*}$ & 0.5 \\
\hline $\mathrm{N} 1$ & $0.27184(9)$ & $0.42428(17)$ & $0.37835(7)$ & $0.0448(5)$ & \\
\hline $\mathrm{N} 2$ & $0.24204(9)$ & $0.36167(17)$ & $0.39907(7)$ & $0.0442(5)$ & \\
\hline N3 & $0.20984(13)$ & $0.3008(3)$ & $0.41775(11)$ & $0.0928(10)$ & \\
\hline $\mathrm{P} 1$ & $0.41536(2)$ & $0.39566(4)$ & $0.33427(2)$ & $0.02831(12)$ & \\
\hline $\mathrm{P} 2$ & $0.35969(3)$ & $0.64007(4)$ & $0.38088(2)$ & $0.03007(12)$ & \\
\hline Ru1 & $0.37307(2)$ & $0.46072(2)$ & $0.40230(2)$ & $0.02988(7)$ & \\
\hline $\mathrm{Cl1}$ & $0.6866(3)$ & $0.9604(3)$ & $0.52581(15)$ & $0.095(2)$ & $0.62(3)$ \\
\hline Cl1A & $0.7012(8)$ & $0.9520(10)$ & $0.5236(4)$ & $0.202(7)$ & $0.38(3)$ \\
\hline
\end{tabular}

Atomic displacement parameters $\left(A^{2}\right)$

\begin{tabular}{lllllll}
\hline & $U^{11}$ & $U^{22}$ & $U^{33}$ & $U^{12}$ & $U^{13}$ & $U^{23}$ \\
\hline C1 & $0.0275(10)$ & $0.0285(10)$ & $0.0335(11)$ & $-0.0023(8)$ & $0.0018(8)$ & $-0.0054(8)$ \\
C2 & $0.0348(11)$ & $0.0401(12)$ & $0.0399(12)$ & $-0.0015(9)$ & $-0.0021(9)$ & $-0.0012(10)$ \\
C3 & $0.0309(11)$ & $0.0516(14)$ & $0.0573(15)$ & $-0.0033(11)$ & $-0.0067(10)$ & $-0.0057(12)$ \\
C4 & $0.0354(13)$ & $0.0510(15)$ & $0.0700(18)$ & $-0.0136(11)$ & $0.0088(12)$ & $-0.0033(13)$ \\
C5 & $0.0481(14)$ & $0.0410(13)$ & $0.0563(15)$ & $-0.0072(11)$ & $0.0080(12)$ & $0.0065(11)$ \\
C6 & $0.0329(11)$ & $0.0338(11)$ & $0.0421(12)$ & $0.0003(9)$ & $0.0019(9)$ & $-0.0028(9)$ \\
C7 & $0.0378(11)$ & $0.0292(11)$ & $0.0349(11)$ & $-0.0014(9)$ & $-0.0065(9)$ & $-0.0007(9)$ \\
C8 & $0.0497(14)$ & $0.0359(12)$ & $0.0526(15)$ & $0.0029(11)$ & $0.0023(11)$ & $-0.0033(11)$ \\
C9 & $0.0625(17)$ & $0.0407(15)$ & $0.077(2)$ & $0.0136(13)$ & $-0.0066(15)$ & $-0.0112(14)$ \\
C10 & $0.096(2)$ & $0.0297(13)$ & $0.078(2)$ & $0.0058(15)$ & $-0.0107(18)$ & $-0.0013(13)$ \\
C11 & $0.087(2)$ & $0.0357(14)$ & $0.091(2)$ & $-0.0197(15)$ & $0.0089(18)$ & $0.0075(15)$ \\
C12 & $0.0510(14)$ & $0.0377(13)$ & $0.0691(18)$ & $-0.0081(11)$ & $0.0071(13)$ & $0.0015(12)$ \\
C13 & $0.0382(11)$ & $0.0249(10)$ & $0.0318(11)$ & $-0.0031(9)$ & $-0.0052(9)$ & $0.0007(8)$ \\
C14 & $0.0462(13)$ & $0.0418(13)$ & $0.0406(13)$ & $-0.0009(10)$ & $-0.0016(10)$ & $-0.0065(10)$ \\
C15 & $0.0780(19)$ & $0.0496(15)$ & $0.0337(13)$ & $-0.0074(13)$ & $-0.0002(12)$ & $-0.0066(11)$ \\
C16 & $0.0781(19)$ & $0.0524(15)$ & $0.0404(14)$ & $-0.0139(14)$ & $-0.0213(13)$ & $-0.0007(12)$ \\
C17 & $0.0486(14)$ & $0.0575(16)$ & $0.0562(17)$ & $-0.0130(12)$ & $-0.0213(12)$ & $0.0006(13)$ \\
C18 & $0.0398(12)$ & $0.0444(13)$ & $0.0411(13)$ & $-0.0081(10)$ & $-0.0060(10)$ & $0.0011(10)$ \\
C19 & $0.0355(11)$ & $0.0340(11)$ & $0.0347(11)$ & $-0.0022(9)$ & $-0.0020(9)$ & $-0.0014(9)$ \\
C20 & $0.0449(13)$ & $0.0444(13)$ & $0.0463(14)$ & $-0.0006(11)$ & $-0.0002(11)$ & $-0.0106(11)$ \\
C21 & $0.0678(18)$ & $0.0505(16)$ & $0.0567(17)$ & $-0.0048(13)$ & $-0.0071(14)$ & $-0.0197(13)$
\end{tabular}




\begin{tabular}{|c|c|c|c|c|c|c|}
\hline $\mathrm{C} 22$ & $0.0559(16)$ & $0.0509(15)$ & $0.0648(18)$ & $-0.0131(13)$ & $-0.0214(13)$ & $-0.0070(13)$ \\
\hline $\mathrm{C} 23$ & $0.0366(13)$ & $0.0532(15)$ & $0.0634(17)$ & $-0.0039(11)$ & $-0.0069(11)$ & $0.0034(13)$ \\
\hline $\mathrm{C} 24$ & $0.0390(12)$ & $0.0425(13)$ & $0.0477(14)$ & $0.0000(10)$ & $-0.0021(10)$ & $-0.0055(11)$ \\
\hline $\mathrm{C} 25$ & $0.0340(11)$ & $0.0439(13)$ & $0.0300(11)$ & $0.0017(10)$ & $0.0020(9)$ & $-0.0062(9)$ \\
\hline $\mathrm{C} 26$ & $0.0478(14)$ & $0.0539(15)$ & $0.0425(13)$ & $0.0109(12)$ & $0.0062(11)$ & $0.0011(11)$ \\
\hline $\mathrm{C} 27$ & $0.0627(17)$ & $0.0670(18)$ & $0.0582(17)$ & $0.0276(15)$ & $0.0039(14)$ & $-0.0034(14)$ \\
\hline $\mathrm{C} 28$ & $0.0520(17)$ & $0.094(2)$ & $0.072(2)$ & $0.0228(17)$ & $0.0162(15)$ & $-0.0151(18)$ \\
\hline $\mathrm{C} 29$ & $0.0668(19)$ & $0.091(2)$ & $0.083(2)$ & $0.0081(18)$ & $0.0440(18)$ & $0.0031(19)$ \\
\hline $\mathrm{C} 30$ & $0.0544(15)$ & $0.0570(16)$ & $0.0567(16)$ & $0.0065(13)$ & $0.0219(13)$ & $0.0033(13)$ \\
\hline C31 & $0.0305(10)$ & $0.0302(10)$ & $0.0318(11)$ & $0.0047(8)$ & $0.0045(8)$ & $-0.0003(8)$ \\
\hline $\mathrm{C} 32$ & $0.0426(13)$ & $0.0443(13)$ & $0.0443(13)$ & $-0.0107(11)$ & $0.0028(10)$ & $0.0006(11)$ \\
\hline $\mathrm{C} 33$ & $0.0496(14)$ & $0.0524(15)$ & $0.0489(15)$ & $-0.0078(12)$ & $0.0133(12)$ & $0.0144(12)$ \\
\hline $\mathrm{C} 34$ & $0.0499(14)$ & $0.0645(16)$ & $0.0341(12)$ & $0.0049(13)$ & $0.0071(11)$ & $0.0131(12)$ \\
\hline $\mathrm{C} 35$ & $0.0494(14)$ & $0.0566(15)$ & $0.0344(12)$ & $-0.0012(12)$ & $-0.0036(10)$ & $0.0010(11)$ \\
\hline $\mathrm{C} 36$ & $0.0389(12)$ & $0.0350(11)$ & $0.0363(12)$ & $-0.0018(9)$ & $0.0023(9)$ & $0.0016(9)$ \\
\hline $\mathrm{C} 37$ & $0.0580(16)$ & $0.102(2)$ & $0.0320(13)$ & $0.0178(17)$ & $0.0087(12)$ & $0.0233(14)$ \\
\hline $\mathrm{C} 38$ & $0.0784(19)$ & $0.0633(17)$ & $0.0244(12)$ & $0.0088(15)$ & $-0.0119(12)$ & $-0.0029(11)$ \\
\hline C39 & $0.0466(14)$ & $0.0661(17)$ & $0.0294(12)$ & $-0.0040(12)$ & $-0.0137(10)$ & $0.0038(11)$ \\
\hline $\mathrm{C} 40$ & $0.0513(14)$ & $0.0582(16)$ & $0.0318(12)$ & $0.0129(12)$ & $-0.0117(10)$ & $0.0097(11)$ \\
\hline $\mathrm{C} 41$ & $0.0657(17)$ & $0.0597(17)$ & $0.0453(15)$ & $-0.0064(14)$ & $-0.0110(13)$ & $0.0264(13)$ \\
\hline $\mathrm{C} 42$ & 0.107 (4) & $0.071(3)$ & $0.103(4)$ & 0 & $0.038(3)$ & 0 \\
\hline N1 & $0.0325(10)$ & $0.0552(12)$ & $0.0459(11)$ & $-0.0045(9)$ & $-0.0029(9)$ & $0.0100(10)$ \\
\hline $\mathrm{N} 2$ & $0.0315(10)$ & $0.0549(13)$ & $0.0459(12)$ & $-0.0017(9)$ & $0.0014(9)$ & $0.0036(10)$ \\
\hline N3 & $0.0596(16)$ & $0.122(2)$ & $0.095(2)$ & $-0.0356(17)$ & $-0.0020(15)$ & $0.0483(19)$ \\
\hline $\mathrm{P} 1$ & 0.0268 & $0.0274(3)$ & $0.0300(3)$ & $-0.0018(2)$ & $-0.0031(2)$ & $-0.0001(2)$ \\
\hline P2 & $0.0296(3)$ & 0.0323 & $0.0282(3)$ & $-0.0013(2)$ & $0.0011(2)$ & $-0.0018(2)$ \\
\hline Ru1 & $0.02908(10)$ & $0.03461(10)$ & $0.02536(10)$ & $-0.00068(7)$ & $-0.00197(6)$ & $0.00396(7)$ \\
\hline $\mathrm{Cl1}$ & $0.0622(18)$ & $0.091(2)$ & $0.137(3)$ & $-0.0075(10)$ & $0.0361(19)$ & $-0.0484(14)$ \\
\hline Cl1A & $0.130(6)$ & $0.165(6)$ & $0.331(15)$ & $-0.006(4)$ & $0.148(9)$ & $-0.068(5)$ \\
\hline
\end{tabular}

Geometric parameters $(\AA, \stackrel{\circ}{)})$

\begin{tabular}{llll}
\hline $\mathrm{C} 1-\mathrm{C} 6$ & $1.389(3)$ & $\mathrm{C} 25-\mathrm{P} 2$ & $1.848(2)$ \\
$\mathrm{C} 1-\mathrm{C} 2$ & $1.397(3)$ & $\mathrm{C} 26-\mathrm{C} 27$ & $1.384(3)$ \\
$\mathrm{C} 1-\mathrm{P} 1$ & $1.833(2)$ & $\mathrm{C} 26-\mathrm{H} 26$ & $1.368(4)$ \\
$\mathrm{C} 2-\mathrm{C} 3$ & $1.379(3)$ & $\mathrm{C} 27-\mathrm{C} 28$ & 0.93 \\
$\mathrm{C} 2-\mathrm{H} 2$ & 0.93 & $\mathrm{C} 27-\mathrm{H} 27$ & $1.359(4)$ \\
$\mathrm{C} 3-\mathrm{C} 4$ & $1.374(4)$ & $\mathrm{C} 28-\mathrm{C} 29$ & 0.93 \\
$\mathrm{C} 3-\mathrm{H} 3$ & 0.93 & $\mathrm{C} 28-\mathrm{H} 28$ & $1.395(4)$ \\
$\mathrm{C} 4-\mathrm{C} 5$ & $1.377(3)$ & $\mathrm{C} 29-\mathrm{C} 30$ & 0.93 \\
$\mathrm{C} 4-\mathrm{H} 4$ & 0.93 & $\mathrm{C} 29-\mathrm{H} 29$ & 0.93 \\
$\mathrm{C} 5-\mathrm{C} 6$ & $\mathrm{C} 30-\mathrm{H} 30$ & $1.384(3)$ \\
$\mathrm{C} 5-\mathrm{H} 5$ & $\mathrm{C} 31-\mathrm{C} 36$ & $1.391(3)$ \\
$\mathrm{C} 6-\mathrm{H} 6$ & $\mathrm{C} 31-\mathrm{C} 32$ & $1.829(2)$ \\
$\mathrm{C} 7-\mathrm{C} 8$ & 0.93 & $\mathrm{C} 31-\mathrm{P} 2$ & $1.384(3)$ \\
$\mathrm{C} 7-\mathrm{C} 12$ & 0.93 & $\mathrm{C} 32-\mathrm{C} 33$ & 0.93 \\
$\mathrm{C} 7-\mathrm{P} 1$ & $1.382(3)$ & $\mathrm{C} 32-\mathrm{H} 32$ & $1.368(3)$ \\
$\mathrm{C} 8-\mathrm{C} 9$ & $1.386(3)$ & $\mathrm{C} 33-\mathrm{C} 34$ &
\end{tabular}




\begin{tabular}{|c|c|c|c|}
\hline $\mathrm{C} 8-\mathrm{H} 8$ & 0.93 & C $33-\mathrm{H} 33$ & 0.93 \\
\hline $\mathrm{C} 9-\mathrm{C} 10$ & $1.362(4)$ & $\mathrm{C} 34-\mathrm{C} 35$ & $1.373(3)$ \\
\hline C9-H9 & 0.93 & C34-H34 & 0.93 \\
\hline $\mathrm{C} 10-\mathrm{C} 11$ & $1.376(4)$ & $\mathrm{C} 35-\mathrm{C} 36$ & $1.378(3)$ \\
\hline $\mathrm{C} 10-\mathrm{H} 10$ & 0.93 & $\mathrm{C} 35-\mathrm{H} 35$ & 0.93 \\
\hline $\mathrm{C} 11-\mathrm{C} 12$ & $1.382(4)$ & C36-H36 & 0.93 \\
\hline $\mathrm{C} 11-\mathrm{H} 11$ & 0.93 & C37-C38 & $1.403(4)$ \\
\hline $\mathrm{C} 12-\mathrm{H} 12$ & 0.93 & $\mathrm{C} 37-\mathrm{C} 41$ & $1.426(4)$ \\
\hline $\mathrm{C} 13-\mathrm{C} 18$ & $1.388(3)$ & C37-Ru1 & $2.207(2)$ \\
\hline $\mathrm{C} 13-\mathrm{C} 14$ & $1.389(3)$ & C $37-\mathrm{H} 37$ & 0.98 \\
\hline $\mathrm{C} 13-\mathrm{P} 1$ & $1.846(2)$ & C $38-\mathrm{C} 39$ & $1.404(4)$ \\
\hline $\mathrm{C} 14-\mathrm{C} 15$ & $1.381(3)$ & $\mathrm{C} 38-\mathrm{Ru} 1$ & $2.193(2)$ \\
\hline $\mathrm{C} 14-\mathrm{H} 14$ & 0.93 & C $38-\mathrm{H} 38$ & 0.98 \\
\hline $\mathrm{C} 15-\mathrm{C} 16$ & $1.375(4)$ & $\mathrm{C} 39-\mathrm{C} 40$ & $1.402(4)$ \\
\hline C15-H15 & 0.93 & C39-Ru1 & $2.177(2)$ \\
\hline $\mathrm{C} 16-\mathrm{C} 17$ & $1.371(4)$ & C $39-\mathrm{H} 39$ & 0.98 \\
\hline $\mathrm{C} 16-\mathrm{H} 16$ & 0.93 & $\mathrm{C} 40-\mathrm{C} 41$ & $1.395(4)$ \\
\hline $\mathrm{C} 17-\mathrm{C} 18$ & $1.389(3)$ & $\mathrm{C} 40-\mathrm{Ru} 1$ & $2.202(2)$ \\
\hline C17-H17 & 0.93 & $\mathrm{C} 40-\mathrm{H} 40$ & 0.98 \\
\hline C18-H18 & 0.93 & $\mathrm{C} 41-\mathrm{Ru} 1$ & $2.211(2)$ \\
\hline $\mathrm{C} 19-\mathrm{C} 20$ & $1.383(3)$ & $\mathrm{C} 41-\mathrm{H} 41$ & 0.98 \\
\hline $\mathrm{C} 19-\mathrm{C} 24$ & $1.395(3)$ & $\mathrm{C} 42-\mathrm{Cl} \mathrm{A}^{\mathrm{i}}$ & $1.508(16)$ \\
\hline $\mathrm{C} 19-\mathrm{P} 2$ & $1.840(2)$ & $\mathrm{C} 42-\mathrm{C} 11 \mathrm{~A}$ & $1.508(16)$ \\
\hline $\mathrm{C} 20-\mathrm{C} 21$ & $1.384(3)$ & $\mathrm{C} 42-\mathrm{Cl1}^{\mathrm{i}}$ & $1.807(7)$ \\
\hline $\mathrm{C} 20-\mathrm{H} 20$ & 0.93 & $\mathrm{C} 42-\mathrm{Cl1}$ & $1.807(7)$ \\
\hline $\mathrm{C} 21-\mathrm{C} 22$ & $1.371(4)$ & $\mathrm{C} 42-\mathrm{H} 42 \mathrm{~A}$ & 0.97 \\
\hline $\mathrm{C} 21-\mathrm{H} 21$ & 0.93 & $\mathrm{C} 42-\mathrm{H} 42 \mathrm{~B}$ & 0.97 \\
\hline $\mathrm{C} 22-\mathrm{C} 23$ & $1.374(4)$ & $\mathrm{N} 1-\mathrm{N} 2$ & $1.173(3)$ \\
\hline $\mathrm{C} 22-\mathrm{H} 22$ & 0.93 & $\mathrm{~N} 1-\mathrm{Ru} 1$ & $2.1553(18)$ \\
\hline $\mathrm{C} 23-\mathrm{C} 24$ & $1.382(3)$ & $\mathrm{N} 2-\mathrm{N} 3$ & $1.156(3)$ \\
\hline $\mathrm{C} 23-\mathrm{H} 23$ & 0.93 & $\mathrm{P} 1-\mathrm{Ru} 1$ & $2.3314(6)$ \\
\hline $\mathrm{C} 24-\mathrm{H} 24$ & 0.93 & $\mathrm{P} 2-\mathrm{Ru} 1$ & $2.3274(6)$ \\
\hline $\mathrm{C} 25-\mathrm{C} 30$ & $1.373(3)$ & $\mathrm{C} 11 \mathrm{~A}-\mathrm{Cl}^{1} \mathrm{~A}^{\mathrm{i}}$ & $2.47(3)$ \\
\hline $\mathrm{C} 25-\mathrm{C} 26$ & $1.389(3)$ & & \\
\hline $\mathrm{C} 6-\mathrm{C} 1-\mathrm{C} 2$ & $117.70(19)$ & $\mathrm{C} 33-\mathrm{C} 32-\mathrm{H} 32$ & 119.9 \\
\hline $\mathrm{C} 6-\mathrm{C} 1-\mathrm{P} 1$ & $121.59(15)$ & $\mathrm{C} 31-\mathrm{C} 32-\mathrm{H} 32$ & 119.9 \\
\hline $\mathrm{C} 2-\mathrm{C} 1-\mathrm{P} 1$ & $120.33(16)$ & $\mathrm{C} 34-\mathrm{C} 33-\mathrm{C} 32$ & $120.5(2)$ \\
\hline $\mathrm{C} 3-\mathrm{C} 2-\mathrm{C} 1$ & $121.3(2)$ & $\mathrm{C} 34-\mathrm{C} 33-\mathrm{H} 33$ & 119.8 \\
\hline $\mathrm{C} 3-\mathrm{C} 2-\mathrm{H} 2$ & 119.4 & $\mathrm{C} 32-\mathrm{C} 33-\mathrm{H} 33$ & 119.8 \\
\hline $\mathrm{C} 1-\mathrm{C} 2-\mathrm{H} 2$ & 119.4 & $\mathrm{C} 33-\mathrm{C} 34-\mathrm{C} 35$ & $119.9(2)$ \\
\hline $\mathrm{C} 4-\mathrm{C} 3-\mathrm{C} 2$ & $120.3(2)$ & $\mathrm{C} 33-\mathrm{C} 34-\mathrm{H} 34$ & 120.1 \\
\hline $\mathrm{C} 4-\mathrm{C} 3-\mathrm{H} 3$ & 119.9 & $\mathrm{C} 35-\mathrm{C} 34-\mathrm{H} 34$ & 120.1 \\
\hline $\mathrm{C} 2-\mathrm{C} 3-\mathrm{H} 3$ & 119.9 & $\mathrm{C} 34-\mathrm{C} 35-\mathrm{C} 36$ & $120.1(2)$ \\
\hline $\mathrm{C} 3-\mathrm{C} 4-\mathrm{C} 5$ & $119.5(2)$ & $\mathrm{C} 34-\mathrm{C} 35-\mathrm{H} 35$ & 120 \\
\hline $\mathrm{C} 3-\mathrm{C} 4-\mathrm{H} 4$ & 120.2 & $\mathrm{C} 36-\mathrm{C} 35-\mathrm{H} 35$ & 120 \\
\hline $\mathrm{C} 5-\mathrm{C} 4-\mathrm{H} 4$ & 120.2 & $\mathrm{C} 35-\mathrm{C} 36-\mathrm{C} 31$ & 120.9 \\
\hline $\mathrm{C} 4-\mathrm{C} 5-\mathrm{C} 6$ & $120.6(2)$ & $\mathrm{C} 35-\mathrm{C} 36-\mathrm{H} 36$ & 119.5 \\
\hline
\end{tabular}




\begin{tabular}{|c|c|c|c|}
\hline $\mathrm{C} 4-\mathrm{C} 5-\mathrm{H} 5$ & 119.7 & $\mathrm{C} 31-\mathrm{C} 36-\mathrm{H} 36$ & 119.5 \\
\hline $\mathrm{C} 6-\mathrm{C} 5-\mathrm{H} 5$ & 119.7 & $\mathrm{C} 38-\mathrm{C} 37-\mathrm{C} 41$ & $107.6(2)$ \\
\hline $\mathrm{C} 5-\mathrm{C} 6-\mathrm{C} 1$ & $120.7(2)$ & $\mathrm{C} 38-\mathrm{C} 37-\mathrm{Ru} 1$ & $70.89(14)$ \\
\hline $\mathrm{C} 5-\mathrm{C} 6-\mathrm{H} 6$ & 119.7 & $\mathrm{C} 41-\mathrm{C} 37-\mathrm{Ru} 1$ & $71.33(14)$ \\
\hline $\mathrm{C} 1-\mathrm{C} 6-\mathrm{H} 6$ & 119.7 & $\mathrm{C} 38-\mathrm{C} 37-\mathrm{H} 37$ & 126.1 \\
\hline $\mathrm{C} 8-\mathrm{C} 7-\mathrm{C} 12$ & $118.4(2)$ & $\mathrm{C} 41-\mathrm{C} 37-\mathrm{H} 37$ & 126.1 \\
\hline $\mathrm{C} 8-\mathrm{C} 7-\mathrm{P} 1$ & $124.26(17)$ & $\mathrm{Ru} 1-\mathrm{C} 37-\mathrm{H} 37$ & 126.1 \\
\hline $\mathrm{C} 12-\mathrm{C} 7-\mathrm{P} 1$ & $117.37(17)$ & $\mathrm{C} 37-\mathrm{C} 38-\mathrm{C} 39$ & $108.4(3)$ \\
\hline $\mathrm{C} 9-\mathrm{C} 8-\mathrm{C} 7$ & $120.8(2)$ & $\mathrm{C} 37-\mathrm{C} 38-\mathrm{Ru} 1$ & $71.92(15)$ \\
\hline $\mathrm{C} 9-\mathrm{C} 8-\mathrm{H} 8$ & 119.6 & $\mathrm{C} 39-\mathrm{C} 38-\mathrm{Ru} 1$ & $70.62(13)$ \\
\hline $\mathrm{C} 7-\mathrm{C} 8-\mathrm{H} 8$ & 119.6 & $\mathrm{C} 37-\mathrm{C} 38-\mathrm{H} 38$ & 125.7 \\
\hline $\mathrm{C} 10-\mathrm{C} 9-\mathrm{C} 8$ & $120.3(3)$ & $\mathrm{C} 39-\mathrm{C} 38-\mathrm{H} 38$ & 125.7 \\
\hline $\mathrm{C} 10-\mathrm{C} 9-\mathrm{H} 9$ & 119.8 & $\mathrm{Ru} 1-\mathrm{C} 38-\mathrm{H} 38$ & 125.7 \\
\hline $\mathrm{C} 8-\mathrm{C} 9-\mathrm{H} 9$ & 119.8 & $\mathrm{C} 40-\mathrm{C} 39-\mathrm{C} 38$ & $107.6(2)$ \\
\hline $\mathrm{C} 9-\mathrm{C} 10-\mathrm{C} 11$ & $119.8(3)$ & $\mathrm{C} 40-\mathrm{C} 39-\mathrm{Ru} 1$ & $72.30(13)$ \\
\hline $\mathrm{C} 9-\mathrm{C} 10-\mathrm{H} 10$ & 120.1 & $\mathrm{C} 38-\mathrm{C} 39-\mathrm{Ru} 1$ & $71.88(13)$ \\
\hline $\mathrm{C} 11-\mathrm{C} 10-\mathrm{H} 10$ & 120.1 & $\mathrm{C} 40-\mathrm{C} 39-\mathrm{H} 39$ & 126.1 \\
\hline $\mathrm{C} 10-\mathrm{C} 11-\mathrm{C} 12$ & $120.2(3)$ & $\mathrm{C} 38-\mathrm{C} 39-\mathrm{H} 39$ & 126.1 \\
\hline $\mathrm{C} 10-\mathrm{C} 11-\mathrm{H} 11$ & 119.9 & $\mathrm{Ru} 1-\mathrm{C} 39-\mathrm{H} 39$ & 126.1 \\
\hline $\mathrm{C} 12-\mathrm{C} 11-\mathrm{H} 11$ & 119.9 & $\mathrm{C} 41-\mathrm{C} 40-\mathrm{C} 39$ & $109.1(2)$ \\
\hline $\mathrm{C} 11-\mathrm{C} 12-\mathrm{C} 7$ & $120.4(3)$ & $\mathrm{C} 41-\mathrm{C} 40-\mathrm{Ru} 1$ & $71.92(13)$ \\
\hline $\mathrm{C} 11-\mathrm{C} 12-\mathrm{H} 12$ & 119.8 & $\mathrm{C} 39-\mathrm{C} 40-\mathrm{Ru} 1$ & $70.34(13)$ \\
\hline $\mathrm{C} 7-\mathrm{C} 12-\mathrm{H} 12$ & 119.8 & $\mathrm{C} 41-\mathrm{C} 40-\mathrm{H} 40$ & 125.4 \\
\hline $\mathrm{C} 18-\mathrm{C} 13-\mathrm{C} 14$ & $118.4(2)$ & $\mathrm{C} 39-\mathrm{C} 40-\mathrm{H} 40$ & 125.4 \\
\hline $\mathrm{C} 18-\mathrm{C} 13-\mathrm{P} 1$ & $119.45(17)$ & $\mathrm{Ru} 1-\mathrm{C} 40-\mathrm{H} 40$ & 125.4 \\
\hline $\mathrm{C} 14-\mathrm{C} 13-\mathrm{P} 1$ & $121.98(16)$ & $\mathrm{C} 40-\mathrm{C} 41-\mathrm{C} 37$ & $107.3(3)$ \\
\hline $\mathrm{C} 15-\mathrm{C} 14-\mathrm{C} 13$ & $120.7(2)$ & $\mathrm{C} 40-\mathrm{C} 41-\mathrm{Ru} 1$ & $71.24(13)$ \\
\hline $\mathrm{C} 15-\mathrm{C} 14-\mathrm{H} 14$ & 119.7 & $\mathrm{C} 37-\mathrm{C} 41-\mathrm{Ru} 1$ & $71.00(14)$ \\
\hline $\mathrm{C} 13-\mathrm{C} 14-\mathrm{H} 14$ & 119.7 & $\mathrm{C} 40-\mathrm{C} 41-\mathrm{H} 41$ & 126.3 \\
\hline $\mathrm{C} 16-\mathrm{C} 15-\mathrm{C} 14$ & $120.4(2)$ & $\mathrm{C} 37-\mathrm{C} 41-\mathrm{H} 41$ & 126.3 \\
\hline $\mathrm{C} 16-\mathrm{C} 15-\mathrm{H} 15$ & 119.8 & $\mathrm{Ru} 1-\mathrm{C} 41-\mathrm{H} 41$ & 126.3 \\
\hline $\mathrm{C} 14-\mathrm{C} 15-\mathrm{H} 15$ & 119.8 & $\mathrm{C} 11 \mathrm{~A}-\mathrm{C} 42-\mathrm{C} 11 \mathrm{~A}$ & $109.8(9)$ \\
\hline $\mathrm{C} 17-\mathrm{C} 16-\mathrm{C} 15$ & $119.6(2)$ & $\mathrm{C} 11^{\mathrm{i}}-\mathrm{C} 42-\mathrm{Cl1}$ & $114.9(4)$ \\
\hline $\mathrm{C} 17-\mathrm{C} 16-\mathrm{H} 16$ & 120.2 & $\mathrm{C} 11^{\mathrm{i}}-\mathrm{C} 42-\mathrm{H} 42 \mathrm{~A}$ & 108.5 \\
\hline $\mathrm{C} 15-\mathrm{C} 16-\mathrm{H} 16$ & 120.2 & $\mathrm{Cl1}-\mathrm{C} 42-\mathrm{H} 42 \mathrm{~A}$ & 108.5 \\
\hline $\mathrm{C} 16-\mathrm{C} 17-\mathrm{C} 18$ & $120.5(2)$ & $\mathrm{C} 11^{\mathrm{i}}-\mathrm{C} 42-\mathrm{H} 42 \mathrm{~B}$ & 108.5 \\
\hline $\mathrm{C} 16-\mathrm{C} 17-\mathrm{H} 17$ & 119.7 & $\mathrm{Cl1}-\mathrm{C} 42-\mathrm{H} 42 \mathrm{~B}$ & 108.5 \\
\hline $\mathrm{C} 18-\mathrm{C} 17-\mathrm{H} 17$ & 119.7 & $\mathrm{H} 42 \mathrm{~A}-\mathrm{C} 42-\mathrm{H} 42 \mathrm{~B}$ & 107.5 \\
\hline $\mathrm{C} 13-\mathrm{C} 18-\mathrm{C} 17$ & $120.3(2)$ & $\mathrm{N} 2-\mathrm{N} 1-\mathrm{Ru} 1$ & $119.20(15)$ \\
\hline $\mathrm{C} 13-\mathrm{C} 18-\mathrm{H} 18$ & 119.8 & $\mathrm{~N} 3-\mathrm{N} 2-\mathrm{N} 1$ & $176.3(3)$ \\
\hline $\mathrm{C} 17-\mathrm{C} 18-\mathrm{H} 18$ & 119.8 & $\mathrm{C} 1-\mathrm{P} 1-\mathrm{C} 13$ & $103.84(9)$ \\
\hline $\mathrm{C} 20-\mathrm{C} 19-\mathrm{C} 24$ & $118.3(2)$ & $\mathrm{C} 1-\mathrm{P} 1-\mathrm{C} 7$ & $104.47(9)$ \\
\hline $\mathrm{C} 20-\mathrm{C} 19-\mathrm{P} 2$ & $123.07(17)$ & $\mathrm{C} 13-\mathrm{P} 1-\mathrm{C} 7$ & $97.68(9)$ \\
\hline $\mathrm{C} 24-\mathrm{C} 19-\mathrm{P} 2$ & $118.47(16)$ & $\mathrm{C} 1-\mathrm{P} 1-\mathrm{Ru} 1$ & $110.94(6)$ \\
\hline $\mathrm{C} 19-\mathrm{C} 20-\mathrm{C} 21$ & $120.3(2)$ & $\mathrm{C} 13-\mathrm{P} 1-\mathrm{Ru} 1$ & $126.90(7)$ \\
\hline $\mathrm{C} 19-\mathrm{C} 20-\mathrm{H} 20$ & 119.8 & $\mathrm{C} 7-\mathrm{P} 1-\mathrm{Ru} 1$ & $110.42(7)$ \\
\hline $\mathrm{C} 21-\mathrm{C} 20-\mathrm{H} 20$ & 119.8 & $\mathrm{C} 31-\mathrm{P} 2-\mathrm{C} 19$ & $102.66(10)$ \\
\hline $\mathrm{C} 22-\mathrm{C} 21-\mathrm{C} 20$ & $120.6(2)$ & $\mathrm{C} 31-\mathrm{P} 2-\mathrm{C} 25$ & $99.05(9)$ \\
\hline
\end{tabular}




\begin{tabular}{|c|c|}
\hline $\mathrm{C} 22-\mathrm{C} 21-\mathrm{H} 21$ & 119.7 \\
\hline $\mathrm{C} 20-\mathrm{C} 21-\mathrm{H} 21$ & 119.7 \\
\hline $\mathrm{C} 21-\mathrm{C} 22-\mathrm{C} 23$ & $120.0(2)$ \\
\hline $\mathrm{C} 21-\mathrm{C} 22-\mathrm{H} 22$ & 120 \\
\hline $\mathrm{C} 23-\mathrm{C} 22-\mathrm{H} 22$ & 120 \\
\hline $\mathrm{C} 22-\mathrm{C} 23-\mathrm{C} 24$ & $119.7(2)$ \\
\hline $\mathrm{C} 22-\mathrm{C} 23-\mathrm{H} 23$ & 120.2 \\
\hline $\mathrm{C} 24-\mathrm{C} 23-\mathrm{H} 23$ & 120.2 \\
\hline $\mathrm{C} 23-\mathrm{C} 24-\mathrm{C} 19$ & $121.0(2)$ \\
\hline $\mathrm{C} 23-\mathrm{C} 24-\mathrm{H} 24$ & 119.5 \\
\hline $\mathrm{C} 19-\mathrm{C} 24-\mathrm{H} 24$ & 119.5 \\
\hline $\mathrm{C} 30-\mathrm{C} 25-\mathrm{C} 26$ & $118.4(2)$ \\
\hline $\mathrm{C} 30-\mathrm{C} 25-\mathrm{P} 2$ & $120.58(18)$ \\
\hline $\mathrm{C} 26-\mathrm{C} 25-\mathrm{P} 2$ & $121.05(17)$ \\
\hline $\mathrm{C} 27-\mathrm{C} 26-\mathrm{C} 25$ & $120.8(2)$ \\
\hline $\mathrm{C} 27-\mathrm{C} 26-\mathrm{H} 26$ & 119.6 \\
\hline $\mathrm{C} 25-\mathrm{C} 26-\mathrm{H} 26$ & 119.6 \\
\hline $\mathrm{C} 28-\mathrm{C} 27-\mathrm{C} 26$ & $120.1(3)$ \\
\hline $\mathrm{C} 28-\mathrm{C} 27-\mathrm{H} 27$ & 119.9 \\
\hline $\mathrm{C} 26-\mathrm{C} 27-\mathrm{H} 27$ & 119.9 \\
\hline $\mathrm{C} 29-\mathrm{C} 28-\mathrm{C} 27$ & $119.6(3)$ \\
\hline $\mathrm{C} 29-\mathrm{C} 28-\mathrm{H} 28$ & 120.2 \\
\hline $\mathrm{C} 27-\mathrm{C} 28-\mathrm{H} 28$ & 120.2 \\
\hline $\mathrm{C} 28-\mathrm{C} 29-\mathrm{C} 30$ & $120.9(3)$ \\
\hline $\mathrm{C} 28-\mathrm{C} 29-\mathrm{H} 29$ & 119.6 \\
\hline $\mathrm{C} 30-\mathrm{C} 29-\mathrm{H} 29$ & 119.6 \\
\hline $\mathrm{C} 25-\mathrm{C} 30-\mathrm{C} 29$ & $120.2(3)$ \\
\hline $\mathrm{C} 25-\mathrm{C} 30-\mathrm{H} 30$ & 119.9 \\
\hline $\mathrm{C} 29-\mathrm{C} 30-\mathrm{H} 30$ & 119.9 \\
\hline $\mathrm{C} 36-\mathrm{C} 31-\mathrm{C} 32$ & $118.4(2)$ \\
\hline $\mathrm{C} 36-\mathrm{C} 31-\mathrm{P} 2$ & $116.65(16)$ \\
\hline $\mathrm{C} 32-\mathrm{C} 31-\mathrm{P} 2$ & $124.88(16)$ \\
\hline $\mathrm{C} 33-\mathrm{C} 32-\mathrm{C} 31$ & $120.2(2)$ \\
\hline $\mathrm{C} 6-\mathrm{C} 1-\mathrm{C} 2-\mathrm{C} 3$ & $-1.4(3)$ \\
\hline $\mathrm{P} 1-\mathrm{C} 1-\mathrm{C} 2-\mathrm{C} 3$ & $-174.44(18)$ \\
\hline $\mathrm{C} 1-\mathrm{C} 2-\mathrm{C} 3-\mathrm{C} 4$ & $0.8(4)$ \\
\hline $\mathrm{C} 2-\mathrm{C} 3-\mathrm{C} 4-\mathrm{C} 5$ & $0.2(4)$ \\
\hline $\mathrm{C} 3-\mathrm{C} 4-\mathrm{C} 5-\mathrm{C} 6$ & $-0.5(4)$ \\
\hline $\mathrm{C} 4-\mathrm{C} 5-\mathrm{C} 6-\mathrm{C} 1$ & $-0.2(4)$ \\
\hline $\mathrm{C} 2-\mathrm{C} 1-\mathrm{C} 6-\mathrm{C} 5$ & $1.1(3)$ \\
\hline $\mathrm{P} 1-\mathrm{C} 1-\mathrm{C} 6-\mathrm{C} 5$ & $174.04(17)$ \\
\hline $\mathrm{C} 12-\mathrm{C} 7-\mathrm{C} 8-\mathrm{C} 9$ & $-3.3(4)$ \\
\hline $\mathrm{P} 1-\mathrm{C} 7-\mathrm{C} 8-\mathrm{C} 9$ & $176.9(2)$ \\
\hline $\mathrm{C} 7-\mathrm{C} 8-\mathrm{C} 9-\mathrm{C} 10$ & $1.3(4)$ \\
\hline $\mathrm{C} 8-\mathrm{C} 9-\mathrm{C} 10-\mathrm{C} 11$ & $1.7(5)$ \\
\hline $\mathrm{C} 9-\mathrm{C} 10-\mathrm{C} 11-\mathrm{C} 12$ & $-2.7(5)$ \\
\hline $\mathrm{C} 10-\mathrm{C} 11-\mathrm{C} 12-\mathrm{C} 7$ & $0.7(5)$ \\
\hline
\end{tabular}

\begin{tabular}{|c|c|}
\hline $\mathrm{C} 19-\mathrm{P} 2-\mathrm{C} 25$ & $101.98(10)$ \\
\hline $\mathrm{C} 31-\mathrm{P} 2-\mathrm{Ru} 1$ & $123.20(7)$ \\
\hline $\mathrm{C} 19-\mathrm{P} 2-\mathrm{Ru} 1$ & $111.41(7)$ \\
\hline $\mathrm{C} 25-\mathrm{P} 2-\mathrm{Ru} 1$ & $115.71(7)$ \\
\hline $\mathrm{N} 1-\mathrm{Ru} 1-\mathrm{C} 39$ & $156.83(9)$ \\
\hline $\mathrm{N} 1-\mathrm{Ru} 1-\mathrm{C} 38$ & $124.58(10)$ \\
\hline $\mathrm{C} 39-\mathrm{Ru} 1-\mathrm{C} 38$ & $37.49(10)$ \\
\hline $\mathrm{N} 1-\mathrm{Ru} 1-\mathrm{C} 40$ & $130.04(9)$ \\
\hline $\mathrm{C} 39-\mathrm{Ru} 1-\mathrm{C} 40$ & $37.35(9)$ \\
\hline $\mathrm{C} 38-\mathrm{Ru} 1-\mathrm{C} 40$ & $62.02(10)$ \\
\hline $\mathrm{N} 1-\mathrm{Ru} 1-\mathrm{C} 37$ & $94.57(10)$ \\
\hline $\mathrm{C} 39-\mathrm{Ru} 1-\mathrm{C} 37$ & $62.60(10)$ \\
\hline $\mathrm{C} 38-\mathrm{Ru} 1-\mathrm{C} 37$ & $37.19(11)$ \\
\hline $\mathrm{C} 40-\mathrm{Ru} 1-\mathrm{C} 37$ & $62.03(10)$ \\
\hline $\mathrm{N} 1-\mathrm{Ru} 1-\mathrm{C} 41$ & $97.36(9)$ \\
\hline $\mathrm{C} 39-\mathrm{Ru} 1-\mathrm{C} 41$ & $62.57(10)$ \\
\hline $\mathrm{C} 38-\mathrm{Ru} 1-\mathrm{C} 41$ & $62.45(11)$ \\
\hline $\mathrm{C} 40-\mathrm{Ru} 1-\mathrm{C} 41$ & $36.85(9)$ \\
\hline $\mathrm{C} 37-\mathrm{Ru} 1-\mathrm{C} 41$ & $37.67(11)$ \\
\hline $\mathrm{N} 1-\mathrm{Ru} 1-\mathrm{P} 2$ & $91.67(6)$ \\
\hline $\mathrm{C} 39-\mathrm{Ru} 1-\mathrm{P} 2$ & $100.63(7)$ \\
\hline $\mathrm{C} 38-\mathrm{Ru} 1-\mathrm{P} 2$ & $88.63(8)$ \\
\hline $\mathrm{C} 40-\mathrm{Ru} 1-\mathrm{P} 2$ & $137.30(7)$ \\
\hline $\mathrm{C} 37-\mathrm{Ru} 1-\mathrm{P} 2$ & $112.95(9)$ \\
\hline $\mathrm{C} 41-\mathrm{Ru} 1-\mathrm{P} 2$ & $149.66(8)$ \\
\hline $\mathrm{N} 1-\mathrm{Ru} 1-\mathrm{P} 1$ & $93.26(6)$ \\
\hline $\mathrm{C} 39-\mathrm{Ru} 1-\mathrm{P} 1$ & $103.99(7)$ \\
\hline $\mathrm{C} 38-\mathrm{Ru} 1-\mathrm{P} 1$ & $141.32(8)$ \\
\hline $\mathrm{C} 40-\mathrm{Ru} 1-\mathrm{P} 1$ & $88.64(7)$ \\
\hline $\mathrm{C} 37-\mathrm{Ru} 1-\mathrm{P} 1$ & $146.86(8)$ \\
\hline $\mathrm{C} 41-\mathrm{Ru} 1-\mathrm{P} 1$ & $109.34(8)$ \\
\hline $\mathrm{P} 2-\mathrm{Ru} 1-\mathrm{P} 1$ & $98.933(19)$ \\
\hline $\mathrm{C} 42-\mathrm{C} 11 \mathrm{~A}-\mathrm{C} 11 \mathrm{~A}^{\mathrm{i}}$ & $35.1(4)$ \\
\hline $\mathrm{C} 37-\mathrm{C} 38-\mathrm{C} 39-\mathrm{C} 40$ & $-1.6(3)$ \\
\hline $\mathrm{Ru} 1-\mathrm{C} 38-\mathrm{C} 39-\mathrm{C} 40$ & $-63.96(15)$ \\
\hline $\mathrm{C} 37-\mathrm{C} 38-\mathrm{C} 39-\mathrm{Ru} 1$ & $62.36(17)$ \\
\hline $\mathrm{C} 38-\mathrm{C} 39-\mathrm{C} 40-\mathrm{C} 41$ & $1.9(3)$ \\
\hline $\mathrm{Ru} 1-\mathrm{C} 39-\mathrm{C} 40-\mathrm{C} 41$ & $-61.81(16)$ \\
\hline $\mathrm{C} 38-\mathrm{C} 39-\mathrm{C} 40-\mathrm{Ru} 1$ & $63.68(15)$ \\
\hline $\mathrm{C} 39-\mathrm{C} 40-\mathrm{C} 41-\mathrm{C} 37$ & $-1.4(3)$ \\
\hline $\mathrm{Ru} 1-\mathrm{C} 40-\mathrm{C} 41-\mathrm{C} 37$ & $-62.25(16)$ \\
\hline $\mathrm{C} 39-\mathrm{C} 40-\mathrm{C} 41-\mathrm{Ru} 1$ & $60.82(16)$ \\
\hline $\mathrm{C} 38-\mathrm{C} 37-\mathrm{C} 41-\mathrm{C} 40$ & $0.4(3)$ \\
\hline $\mathrm{Ru} 1-\mathrm{C} 37-\mathrm{C} 41-\mathrm{C} 40$ & $62.40(16)$ \\
\hline $\mathrm{C} 38-\mathrm{C} 37-\mathrm{C} 41-\mathrm{Ru} 1$ & $-61.98(17)$ \\
\hline $\mathrm{C} 6-\mathrm{C} 1-\mathrm{P} 1-\mathrm{C} 13$ & 35.67 (19) \\
\hline $\mathrm{C} 2-\mathrm{C} 1-\mathrm{P} 1-\mathrm{C} 13$ & $-151.58(17)$ \\
\hline
\end{tabular}




\begin{tabular}{|c|c|c|c|}
\hline $\mathrm{C} 8-\mathrm{C} 7-\mathrm{C} 12-\mathrm{C} 11$ & $2.3(4)$ & $\mathrm{C} 6-\mathrm{C} 1-\mathrm{P} 1-\mathrm{C} 7$ & $137.55(17)$ \\
\hline $\mathrm{P} 1-\mathrm{C} 7-\mathrm{C} 12-\mathrm{C} 11$ & $-177.9(2)$ & $\mathrm{C} 2-\mathrm{C} 1-\mathrm{P} 1-\mathrm{C} 7$ & $-49.71(19)$ \\
\hline $\mathrm{C} 18-\mathrm{C} 13-\mathrm{C} 14-\mathrm{C} 15$ & $2.0(3)$ & $\mathrm{C} 6-\mathrm{C} 1-\mathrm{P} 1-\mathrm{Ru} 1$ & $-103.47(17)$ \\
\hline $\mathrm{P} 1-\mathrm{C} 13-\mathrm{C} 14-\mathrm{C} 15$ & $177.42(18)$ & $\mathrm{C} 2-\mathrm{C} 1-\mathrm{P} 1-\mathrm{Ru} 1$ & $69.28(18)$ \\
\hline $\mathrm{C} 13-\mathrm{C} 14-\mathrm{C} 15-\mathrm{C} 16$ & $-0.2(4)$ & $\mathrm{C} 18-\mathrm{C} 13-\mathrm{P} 1-\mathrm{C} 1$ & $-152.65(17)$ \\
\hline $\mathrm{C} 14-\mathrm{C} 15-\mathrm{C} 16-\mathrm{C} 17$ & $-1.3(4)$ & $\mathrm{C} 14-\mathrm{C} 13-\mathrm{P} 1-\mathrm{C} 1$ & $31.9(2)$ \\
\hline $\mathrm{C} 15-\mathrm{C} 16-\mathrm{C} 17-\mathrm{C} 18$ & $1.1(4)$ & $\mathrm{C} 18-\mathrm{C} 13-\mathrm{P} 1-\mathrm{C} 7$ & $100.32(18)$ \\
\hline $\mathrm{C} 14-\mathrm{C} 13-\mathrm{C} 18-\mathrm{C} 17$ & $-2.1(3)$ & $\mathrm{C} 14-\mathrm{C} 13-\mathrm{P} 1-\mathrm{C} 7$ & $-75.09(19)$ \\
\hline $\mathrm{P} 1-\mathrm{C} 13-\mathrm{C} 18-\mathrm{C} 17$ & $-177.72(18)$ & $\mathrm{C} 18-\mathrm{C} 13-\mathrm{P} 1-\mathrm{Ru} 1$ & $-22.5(2)$ \\
\hline $\mathrm{C} 16-\mathrm{C} 17-\mathrm{C} 18-\mathrm{C} 13$ & $0.6(4)$ & $\mathrm{C} 14-\mathrm{C} 13-\mathrm{P} 1-\mathrm{Ru} 1$ & $162.12(15)$ \\
\hline $\mathrm{C} 24-\mathrm{C} 19-\mathrm{C} 20-\mathrm{C} 21$ & $-0.3(4)$ & $\mathrm{C} 8-\mathrm{C} 7-\mathrm{P} 1-\mathrm{C} 1$ & $-11.1(2)$ \\
\hline $\mathrm{P} 2-\mathrm{C} 19-\mathrm{C} 20-\mathrm{C} 21$ & $175.5(2)$ & $\mathrm{C} 12-\mathrm{C} 7-\mathrm{P} 1-\mathrm{C} 1$ & $169.00(18)$ \\
\hline $\mathrm{C} 19-\mathrm{C} 20-\mathrm{C} 21-\mathrm{C} 22$ & $0.0(4)$ & $\mathrm{C} 8-\mathrm{C} 7-\mathrm{P} 1-\mathrm{C} 13$ & $95.4(2)$ \\
\hline $\mathrm{C} 20-\mathrm{C} 21-\mathrm{C} 22-\mathrm{C} 23$ & $0.4(4)$ & $\mathrm{C} 12-\mathrm{C} 7-\mathrm{P} 1-\mathrm{C} 13$ & $-84.49(19)$ \\
\hline $\mathrm{C} 21-\mathrm{C} 22-\mathrm{C} 23-\mathrm{C} 24$ & $-0.7(4)$ & $\mathrm{C} 8-\mathrm{C} 7-\mathrm{P} 1-\mathrm{Ru} 1$ & $-130.47(18)$ \\
\hline $\mathrm{C} 22-\mathrm{C} 23-\mathrm{C} 24-\mathrm{C} 19$ & $0.4(4)$ & $\mathrm{C} 12-\mathrm{C} 7-\mathrm{P} 1-\mathrm{Ru} 1$ & 49.67 (19) \\
\hline $\mathrm{C} 20-\mathrm{C} 19-\mathrm{C} 24-\mathrm{C} 23$ & $0.1(3)$ & $\mathrm{C} 36-\mathrm{C} 31-\mathrm{P} 2-\mathrm{C} 19$ & $179.14(16)$ \\
\hline $\mathrm{P} 2-\mathrm{C} 19-\mathrm{C} 24-\mathrm{C} 23$ & $-175.91(19)$ & $\mathrm{C} 32-\mathrm{C} 31-\mathrm{P} 2-\mathrm{C} 19$ & $-4.2(2)$ \\
\hline $\mathrm{C} 30-\mathrm{C} 25-\mathrm{C} 26-\mathrm{C} 27$ & $0.4(4)$ & $\mathrm{C} 36-\mathrm{C} 31-\mathrm{P} 2-\mathrm{C} 25$ & $-76.31(17)$ \\
\hline $\mathrm{P} 2-\mathrm{C} 25-\mathrm{C} 26-\mathrm{C} 27$ & $-179.1(2)$ & $\mathrm{C} 32-\mathrm{C} 31-\mathrm{P} 2-\mathrm{C} 25$ & $100.35(19)$ \\
\hline $\mathrm{C} 25-\mathrm{C} 26-\mathrm{C} 27-\mathrm{C} 28$ & $-0.4(4)$ & $\mathrm{C} 36-\mathrm{C} 31-\mathrm{P} 2-\mathrm{Ru} 1$ & $52.69(18)$ \\
\hline $\mathrm{C} 26-\mathrm{C} 27-\mathrm{C} 28-\mathrm{C} 29$ & $0.0(5)$ & $\mathrm{C} 32-\mathrm{C} 31-\mathrm{P} 2-\mathrm{Ru} 1$ & $-130.65(17)$ \\
\hline $\mathrm{C} 27-\mathrm{C} 28-\mathrm{C} 29-\mathrm{C} 30$ & $0.3(5)$ & $\mathrm{C} 20-\mathrm{C} 19-\mathrm{P} 2-\mathrm{C} 31$ & $114.6(2)$ \\
\hline $\mathrm{C} 26-\mathrm{C} 25-\mathrm{C} 30-\mathrm{C} 29$ & $-0.1(4)$ & $\mathrm{C} 24-\mathrm{C} 19-\mathrm{P} 2-\mathrm{C} 31$ & $-69.56(19)$ \\
\hline $\mathrm{P} 2-\mathrm{C} 25-\mathrm{C} 30-\mathrm{C} 29$ & $179.4(2)$ & $\mathrm{C} 20-\mathrm{C} 19-\mathrm{P} 2-\mathrm{C} 25$ & $12.4(2)$ \\
\hline $\mathrm{C} 28-\mathrm{C} 29-\mathrm{C} 30-\mathrm{C} 25$ & $-0.2(5)$ & $\mathrm{C} 24-\mathrm{C} 19-\mathrm{P} 2-\mathrm{C} 25$ & $-171.83(18)$ \\
\hline $\mathrm{C} 36-\mathrm{C} 31-\mathrm{C} 32-\mathrm{C} 33$ & $-0.2(3)$ & $\mathrm{C} 20-\mathrm{C} 19-\mathrm{P} 2-\mathrm{Ru} 1$ & $-111.66(19)$ \\
\hline $\mathrm{P} 2-\mathrm{C} 31-\mathrm{C} 32-\mathrm{C} 33$ & $-176.82(18)$ & $\mathrm{C} 24-\mathrm{C} 19-\mathrm{P} 2-\mathrm{Ru} 1$ & $64.14(19)$ \\
\hline $\mathrm{C} 31-\mathrm{C} 32-\mathrm{C} 33-\mathrm{C} 34$ & $-1.7(4)$ & $\mathrm{C} 30-\mathrm{C} 25-\mathrm{P} 2-\mathrm{C} 31$ & $145.2(2)$ \\
\hline $\mathrm{C} 32-\mathrm{C} 33-\mathrm{C} 34-\mathrm{C} 35$ & $1.6(4)$ & $\mathrm{C} 26-\mathrm{C} 25-\mathrm{P} 2-\mathrm{C} 31$ & $-35.4(2)$ \\
\hline $\mathrm{C} 33-\mathrm{C} 34-\mathrm{C} 35-\mathrm{C} 36$ & $0.5(4)$ & $\mathrm{C} 30-\mathrm{C} 25-\mathrm{P} 2-\mathrm{C} 19$ & $-109.7(2)$ \\
\hline $\mathrm{C} 34-\mathrm{C} 35-\mathrm{C} 36-\mathrm{C} 31$ & $-2.4(4)$ & $\mathrm{C} 26-\mathrm{C} 25-\mathrm{P} 2-\mathrm{C} 19$ & $69.8(2)$ \\
\hline $\mathrm{C} 32-\mathrm{C} 31-\mathrm{C} 36-\mathrm{C} 35$ & $2.2(3)$ & $\mathrm{C} 30-\mathrm{C} 25-\mathrm{P} 2-\mathrm{Ru} 1$ & $11.4(2)$ \\
\hline $\mathrm{P} 2-\mathrm{C} 31-\mathrm{C} 36-\mathrm{C} 35$ & $179.13(18)$ & $\mathrm{C} 26-\mathrm{C} 25-\mathrm{P} 2-\mathrm{Ru} 1$ & $-169.16(16)$ \\
\hline $\mathrm{C} 41-\mathrm{C} 37-\mathrm{C} 38-\mathrm{C} 39$ & $0.7(3)$ & $\mathrm{Cl} 1^{\mathrm{i}}-\mathrm{C} 42-\mathrm{Cl1} \mathrm{A}-\mathrm{Cl}^{\mathrm{i}} \mathrm{A}^{\mathrm{i}}$ & $3.8(7)$ \\
\hline $\mathrm{Ru} 1-\mathrm{C} 37-\mathrm{C} 38-\mathrm{C} 39$ & $-61.54(16)$ & $\mathrm{Cl} 1-\mathrm{C} 42-\mathrm{Cl} 1 \mathrm{~A}-\mathrm{Cl} \mathrm{A}^{\mathrm{i}}$ & $-126(9)$ \\
\hline $\mathrm{C} 41-\mathrm{C} 37-\mathrm{C} 38-\mathrm{Ru} 1$ & $62.26(17)$ & & \\
\hline
\end{tabular}

Symmetry code: (i) $-x+3 / 2, y,-z+1$.

Hydrogen-bond geometry $\left(A,{ }^{\circ}\right)$

\begin{tabular}{lllll}
\hline$D-\mathrm{H} \cdots A$ & $D-\mathrm{H}$ & $\mathrm{H} \cdots A$ & $D \cdots A$ & $D-\mathrm{H} \cdots A$ \\
\hline $\mathrm{C} 18-\mathrm{H} 18 \cdots \mathrm{N} 1$ & 0.93 & 2.35 & $3.204(3)$ & 153 \\
$\mathrm{C} 23-\mathrm{H} 23 \cdots \mathrm{N} 3^{\mathrm{ii}}$ & 0.93 & 2.62 & $3.537(4)$ & 167 \\
$\mathrm{C} 42-\mathrm{H} 42 A \cdots \mathrm{N} 3^{\mathrm{iii}}$ & 0.97 & 2.4 & $3.338(4)$ & 162 \\
$\mathrm{C} 42-\mathrm{H} 42 B \cdots \mathrm{N} 3^{\mathrm{ii}}$ & 0.97 & 2.4 & $3.338(4)$ & 162 \\
\hline
\end{tabular}

Symmetry codes: (ii) $x+1 / 2,-y+1, z$; (iii) $-x+1,-y+1,-z+1$. 